\title{
Inhibition of Tumor Progression Locus 2 Protein Kinase Suppresses Receptor Activator of Nuclear Factor- $\kappa B$ Ligand-Induced Osteoclastogenesis through Down-Regulation of the c-Fos and Nuclear Factor of Activated T Cells c1 Genes
}

\author{
Kazuya Hirata, $\left.,{ }^{*}, \#, \#, 1\right)$ Hirofumi Taki, ${ }^{a}$ Kouichiro Shinoda, ${ }^{a}$ Hiroyuki Hounoki, ${ }^{a}$ Tatsuro MiYahara, ${ }^{a}$ \\ Kazuyuki Tobe, ${ }^{a}$ Hirofumi Ogawa, ${ }^{b, \#}$ Hisashi Mori, ${ }^{b}$ and Eiji Sugryama ${ }^{a, 2)}$ \\ ${ }^{a}$ Department of Internal Medicine, Faculty of Medicine, University of Toyama; and ${ }^{b}$ Department of Molecular \\ Neuroscience, Faculty of Medicine, University of Toyama; 2630 Sugitani, Toyama 930-0194, Japan. \\ Received June 24, 2009; accepted October 8, 2009; published online October 21, 2009
}

\begin{abstract}
Whether tumor progression locus 2 (Tpl2)/cancer Osaka thyroid (Cot) protein kinase participates in osteoclastogenesis from receptor activator of nuclear factor- $\kappa B$ ligand (RANKL)-stimulated monocytes/macrophages remains elusive. To clarify this, a selective and potent inhibitor of Tpl2, 1,7-naphtyridine-3-carbonitrile, was used. When RAW264.7 cells were stimulated with RANKL, Tpl2 was found to be activated. Under this condition, the Tpl2 inhibitor suppressed osteoclastogenesis in a dose-dependent manner. This was due to the blockade of the phosphorylation of mitogen activated protein kinase/ERK kinase (MEK) and extracellular signal-regulated kinase (ERK), but not c-Jun N-terminal kinase (JNK) or p38, concomitant with the down-regulation of the c-Fos and nuclear factor of activated $T$ cells (NFAT)c1 genes. A long period of RANKL-stimulated cell exposure to the inhibitor suppressed osteoclastogenesis as assessed by tartrate-resistant acid phosphatase (TRAP) staining and pit formation on dentin slices. Almost identical results were obtained with macrophage colony-stimulating factor (M-CSF) and RANKL-stimulated bone marrow cells. These findings suggest the possibility that Tpl2 plays a pivotal role in osteoclastogenesis and thus that its inhibitor is useful for investigating the differentiation of monocytes/macrophages to osteoclasts after treatment with RANKL or other stimuli.
\end{abstract}

Key words tumor progression locus 2 ; receptor activator of nuclear factor- $\kappa \mathrm{B}$ ligand; c-Fos; nuclear factor of activated $\mathrm{T}$ cells c1; osteoclastogenesis

Rheumatoid arthritis (RA) is characterized by the presence of inflammatory synovitis accompanied by the destruction of joint cartilage and bone. ${ }^{3)}$ An increasing body of evidence has demonstrated that osteoclasts are the principal cell type responsible for bone resorption in inflammatory joint diseases. Multinucleated giant cells with the phenotypic features of osteoclasts are present at erosion sites in RA and collagen-induced arthritis animal models. Furthermore, it has been reported that mice lacking osteoclasts were resistant to arthritis-induced bone erosion. ${ }^{4)}$ These findings indicate that exploitation of chemical compounds that inhibit the generation of osteoclasts at inflammatory sites would be useful for the treatment of RA.

Macrophage colony-stimulating factor (M-CSF) and receptor for activation of nuclear factor- $\kappa \mathrm{B}$ ligand (RANKL) are required for osteoclastogenesis from monocytes. ${ }^{5,6)}$ The binding of RANKL to its receptor RANK recruits TNF receptor associated factor 6 (TRAF6) in order to cause sequential downstream events such as the up-regulation of nuclear factor- $\kappa \mathrm{B}(\mathrm{NF}-\kappa \mathrm{B}), \mathrm{p} 38$, c-Jun N-terminal kinase (JNK), extrallular signal-regulated kinase (ERK), AP-1, nuclear factor of activated T cells (NFAT)c1, which activate related genes to induce the differentiation of monocytes into osteoclasts. ${ }^{6,7)}$ Among them, NFATc1 is the most important transcription factor during osteoclastogenesis because NFATc1-deficient embryonic stem cells fail to differentiate into osteoclasts. ${ }^{8,9}$ The expression of c-Fos, a component of AP-1, is also associated with osteoclastogenesis because c-Fos-null mice develop severe osteopetrosis due to a lack of osteoclasts, and no NFATc1 expression is seen in the osteoclast precursors of these animals. ${ }^{6}{ }^{68}$ However, the mechanisms by which the cFos and NFATc1 genes are activated downstream of the RANK-TRAF6 signaling pathway triggered by RANKL have not been fully elucidated.

In this study, we focused on tumor progression locus 2 (Tpl2)/cancer Osaka thyroid (Cot), which is a serine/threonine protein kinase and a member of the mitogen-activated protein kinase kinase kinase (MAP3K) family. ${ }^{10,11)}$ When Tpl2-deficient mice were treated with lipopolysaccharide (LPS), the activation of MEK and ERK, but not JNK or p38 MAP kinases or NF- $\kappa \mathrm{B}$, was abolished, resulting in decreased levels of tumor necrosis factor $\alpha(\mathrm{TNF} \alpha)$ and enhanced resistance to endotoxin shock after LPS/D-galactosamine treatments. ${ }^{11)}$ Moreover, Tpl2 ablation protected the mice from TNF $\alpha$-induced inflammatory bowel disease ${ }^{12)}$ and pancreatic and pulmonary inflammation. ${ }^{13)}$ These results imply that Tp12/Cot plays another role in signal transduction during osteoclast formation. Here, we examined the effects of a recently synthesized Tpl2/Cot inhibitor, 1,7-naphtyridine-3-carbonitrile, ${ }^{14)}$ on RANKL-induced osteoclast formation in the RAW264.7 cell line and M-CSF and RANKLstimulated mouse bone marrow cells. To the best of our knowledge, this is the first report showing that the MAP kinase pathway mediated via Tpl2/Cot plays an important role in RANKL-induced osteoclastogenesis from the monocyte/ macrophage lineage.

\section{MATERIALS AND METHODS}

Materials A Tpl2 inhibitor, 4-(3-chloro-4-fluorophenyl- 
amino)-6-(pyridine-3-yl-methylamino)-3-cyano-[1,7]naphtyridine was purchased from Calbiochem. Recombinant human soluble RANKL and M-CSF were obtained from Pepro Tech. Recombinant full-length GST-MEK1 was from Millipore. The antibodies used were from the companies indicated in parentheses: anti-Tpl2, anti-phosphorylated MEK1/2, anti-c-Fos, anti-NFATc1, anti-glyceraldehyde-3phosphate dehydrogenase (GAPDH) antibodies (Santa Cruz Biotechnology); anti-ERK1/2, anti-phosphorylated ERK1/2, anti-p38, anti-phosphorylated p38, anti-JNK, anti-phosphorylated JNK antibodies (Cell Signaling); anti-MEK1/2 antibody (Transduction Laboratories). The chemical luminescence kit was from Thermo Scientific.

Culture of RAW264.7 Cells This cell line was obtained from the American Type Culture Collection. The cells were spread in a $10-\mathrm{cm}$ dish at a density of $2 \times 10^{6}$ cells/dish and maintained in Dulbecco's modified Eagle's medium (DMEM) (Gibco BRL) supplemented with 10\% fetal bovine serum, penicillin (100 units $/ \mathrm{ml})$, and streptomycin $(100 \mu \mathrm{g} / \mathrm{ml})$ under $5 \% \mathrm{CO}_{2}$ at $37^{\circ} \mathrm{C}$.

Isolation of Murine Bone Marrow Cells Precursors Bone marrow cells were isolated from the femora and tibiae of 7-week-old ddY male mice (Sankyo Labo Service). The bone ends were dissected, and the bone marrow cavity was flushed out into a plastic dish by slowly injecting a minimum essential medium ( $\alpha$-MEM) using a sterile 26-gauge needle. After brief centrifugation, the cell pellet was resuspended in the medium together with $10 \mathrm{ng} / \mathrm{ml}$ of $\mathrm{M}-\mathrm{CSF}$, and the cells were cultured for $24 \mathrm{~h}$. Non-adherent cells were collected and used as bone marrow precursors.

Osteoclastogenesis RAW264.7 cells were spread at $1.5 \times 10^{3}$ cells/well in 96-well plates and cultured in DMEM supplemented with RANKL $(100 \mathrm{ng} / \mathrm{ml})$ for $6 \mathrm{~d}$. Various concentrations of the Tpl2 inhibitor were added $3 \mathrm{~d}$ before the end of the culture. The culture medium was replaced with a fresh medium containing the supplements every $3 \mathrm{~d}$. Similarly, bone marrow precursors were plated at $1.5 \times 10^{5}$ cells/well in 96-well plates and cultured with $\alpha$-MEM containing M-CSF $(10 \mathrm{ng} / \mathrm{ml})$ and RANKL $(100 \mathrm{ng} / \mathrm{ml})$ for $7 \mathrm{~d}$. Various concentrations of the Tpl2 inhibitor were added $3 \mathrm{~d}$ before the end of the culture. The culture medium was replaced with fresh medium on days 3 and 5 after the first RANKL stimulation.

Tpl2 Kinase Activity Assay The Tpl2 kinase activity was estimated by measuring the phosphorylation level of MEK1 by Western blotting. ${ }^{15)}$ Five-hundred $\mu \mathrm{g}$ cell lysate samples from 6 -well plates were incubated with $2 \mu \mathrm{g}$ of antiTpl2 antibody at $4{ }^{\circ} \mathrm{C}$ for $4 \mathrm{~h}$ and with $20 \mu \mathrm{l}$ of protein $\mathrm{A} / \mathrm{G}$ agarose beads (Santa Cruz Biotechnology) for another $1 \mathrm{~h}$. After centrifugation, the precipitate was reacted with $1 \mu \mathrm{g}$ of the substrate GST-MEK1 (Millipore) in a $40 \mu$ l solution consisting of $50 \mathrm{~mm}$ Tris-acetate $(\mathrm{pH} 7.5), 0.1 \mathrm{~mm}$ ethylene glycol bis(2-aminoethyl ether)- $N, N, N^{\prime}, N^{\prime}$-tetraacetic acid (EGTA), $0.1 \mathrm{~mm}$ sodium orthovanadate, $10 \mathrm{~mm}$ magnesium acetate, $200 \mu \mathrm{M}$ ATP, $0.1 \%$ 2-mercaptoethanol, and $0.03 \%$ Brij 35 at $30^{\circ} \mathrm{C}$ for $30 \mathrm{~min}$.

Quantitative Reverse-Transcription and PolymeraseChain Reaction (qRT-PCR) Total RNA was extracted from the treated cells with an RNeasy ${ }^{\mathrm{TM}}$ kit (Qiagen). Fivehundred ng of total RNA were reverse-transcribed to cDNA using a PrimeScript $\mathrm{RT}^{\mathrm{TM}}$ reagent kit (Takara Bio). qRT-PCR was carried out using a combination of a SYBR premix EX Taq II $^{\mathrm{TM}}$ (Takara Bio) with an $\mathrm{Mx} 3000 \mathrm{P}^{\mathrm{TM}}$ real-time PCR system (Stratagene). The PCR primers were as follows: NFATc1 sense primer: 5'-TCA TCC TGT CCA ACA CCA AA-3'; NFATc1 anti-sense primer: 5'-TCA CCC TGG TGT TCT TCC TC-3'; c-Fos sense primer: 5'-ATG GGC TCT CCT GTC AAC AC-3'; c-Fos anti-sense primer: 5'-TCT ACT TTG CCC CTT CTG CC-3'; GAPDH sense primer: 5' TGA AGC AGG CAT CTG AGG G-3'; GAPDH anti-sense primer: 5'-CGA AGG TGG AAG AGT GGG AG-3'. The PCR conditions were as follows: $95^{\circ} \mathrm{C}$ for $10 \mathrm{~s}$ for initial denaturation and 40 cycles of denaturation at $95^{\circ} \mathrm{C}$ for $5 \mathrm{~s}$ followed by annealing/elongation at $55^{\circ} \mathrm{C}$ for $35 \mathrm{~s}$. GAPDH was used as an internal control.

Tartrate-Resistant Acid Phosphatase (TRAP) Staining To determine the number of osteoclast cells, the TRAP staining method was used. ${ }^{16)}$ Briefly, the cultured cells were successively treated with $10 \%$ formaldehyde $(\mathrm{v} / \mathrm{v})$ in PBS $(-)$ for $10 \mathrm{~min}$, an equal volume of ethanol/acetone for $1 \mathrm{~min}$, and the TRAP-staining solution at room temperature for about $30 \mathrm{~min}$. The TRAP-positive cells appeared dark red. The TRAP-positive and multinucleated cells that contained three or more nuclei were judged to be osteoclasts.

Pit Formation on Dentine Osteoclast bone resorption was assessed by the pit formation assay. ${ }^{16)}$ RAW264.7 cells $\left(1 \times 10^{3}\right.$ cells/well) were placed on dentin slices (4-mm square) in a 96-well plate and cultured with RANKL $(100 \mathrm{ng} / \mathrm{ml})$ for $10 \mathrm{~d}$. Various concentrations of the Tpl2 inhibitor were added $7 \mathrm{~d}$ before at the end of the culture. The culture medium was replaced with fresh medium every $3 \mathrm{~d}$. Similarly, bone marrow precursors were plated at $1.5 \times 10^{5}$ cells/well and cultured with M-CSF (10 ng/ml) and RANKL $(100 \mathrm{ng} / \mathrm{ml})$ for $7 \mathrm{~d}$. Various concentrations of the Tpl2 inhibitor were added $3 \mathrm{~d}$ before at the end of the culture. The culture medium was replaced with fresh medium on days 3 and 5 after the first RANKL stimulation. Then, the slices were left in $1 \mathrm{~m}$ ammonium hydroxide to remove the attached cells, stained with toluidine blue $(0.1 \%)$ in borax solution $(0.1 \%)$, and subjected to photography under light microscopy.

Other Methods The procedures for Western blot analysis were described previously. ${ }^{16)}$ Cell viability was monitored after the addition of the inhibitor by $0.3 \%$ trypan blue staining or by measuring lactate dehydrogenase activity in the medium using a cytotoxicity detection kit (Roche).

\section{RESULTS AND DISCUSSION}

Tpl2 Inhibitor Markedly Suppresses RANKL-Induced Osteoclastogenesis The naphthyridine-3-carbonitriles, which are Tpl2 inhibitors, were initially explored as ATP binding competitors of protein kinases. ${ }^{14)}$ This series of compounds are reported to be quite specific for $\mathrm{Tp} 12$ with $\mathrm{IC}_{50}$ of $12-50 \mathrm{~nm}$. This affinity is at least 2000- to 10000-fold higher than those for other protein kinases such as Raf-1, MEK, p38, CaMKII, and so on. ${ }^{17)}$ Before the use of the inhibitor, we first wanted to know whether the signal induced by RANKL stimulation in RAW264.7 cells is transmitted to Tp12. To achieve this, Tpl2 protein kinase activity was assessed by measuring the cell-free phosphorylation of MEK1 as a substrate. Figure 1A shows that the Tpl2 levels were un- 

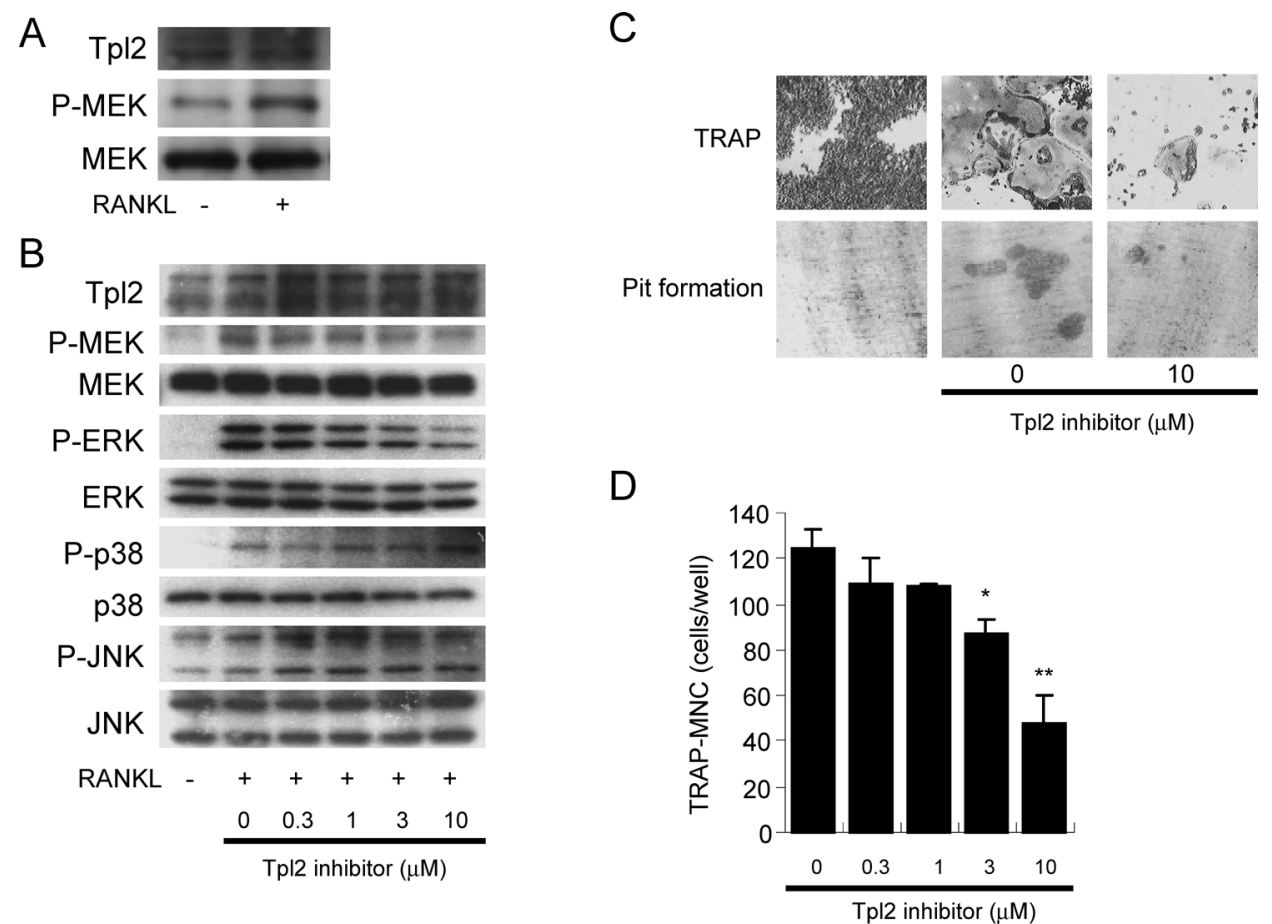

Fig. 1. Suppression of RANKL-Induced Phosphorylation of the MEK-ERK Signaling Pathway and Osteoclastogenesis by the Tp12 Inhibitor

(A) RAW264.7 cells were stimulated with RANKL ( $100 \mathrm{ng} / \mathrm{ml}$ ) for $30 \mathrm{~min}$, and then Tpl2 kinase activity was measured as described in Materials and Methods. (B) RAW264.7 cells were incubated with various concentrations of Tpl2 inhibitor for $1 \mathrm{~h}$ and then exposed to RANKL ( $100 \mathrm{ng} / \mathrm{ml}$ ) for an additional $30 \mathrm{~min}$. The phosphorylated and unphosphorylated forms of each protein were visualized by Western blotting as described in Materials and Methods. (C) RAW264.7 cells were differentiated into osteoclasts in the presence or absence of the Tpl2 inhibitor as described in Materials and Methods. The cells were subjected to TRAP staining (upper panel). Separately, these cells were processed by the pit formation assay on dentin slices (lower panel). (D) The number of TRAP-positive multinuclear cells (TRAP-MNCs) was quantified. Values are shown as the means + S.D. of three independent experiments. $* p<0.01$, and $* * p<0.001$ compared with the results in the absence of the Tpl2 inhibitor, according to the Students' $t$-test.

changed between extracts from the RANKL-stimulated and control cells, while MEK1 was markedly phosphorylated in the RANKL-stimulated extract compared with the control extract. This result indicates that signals triggered by RANKL stimulation are transmitted to MEK1 via Tpl2 activation. Then, we examined whether the Tpl2 inhibitor was able to block the MAPK signaling pathway. As shown in Fig. 1B, RANKL-stimulation induced the phosphorylation of MEK, ERK, p38, and JNK from 1.5 to 2-fold. The Tpl2 inhibitor dose-dependently inhibited the induction of MEK and ERK but not p38 or JNK. This result indicates that the Tp12 inhibitor blocks the Tpl2-MEK-ERK pathway in RANKLstimulated cells. Thus, we next examined how the Tpl2 inhibitor affects RANKL-induced osteoclastogenesis. As shown in Fig. 1C (upper panel), the number of TRAP-positive multinuclear cells was decreased by the Tpl2 inhibitor. The formation of osteoclasts was also verified by a pit formation assay (lower panel). The Tpl2 inhibitor was found to block the absorption of the dentin slice. Quantification of this suppression is shown in Fig. 1D. At concentrations exceeding $3 \mu \mathrm{M}$ of the Tpl2 inhibitor, the inhibitory effect became prominent. Even under these conditions, cell viability was not affected as revealed by trypan blue staining and the lactate dehydrogenase release assay (not shown).

Tp12 Inhibitor Profoundly Blocks Induction of the Transcription Factors c-Fos and NFATc1 in RANKLTreated Cells Recent studies have shown that c-Fos and NFATc1 play essential roles in osteoclast differentiation from RANKL-stimulated RAW264.7 cells and bone marrow cells. $^{6,8,9)}$ Hence, we addressed whether the inhibitory effect of Tpl2 is associated with the gene expression of NFATc1 and c-Fos. As shown in Fig. 2A, whereas both c-Fos and NFATc1 mRNA levels were augmented about 3-fold by RANKL treatment, these increases were abolished by raising the Tpl2 concentration. Likewise, the increases in the c-Fos and NFATc1 protein levels induced by RANKL stimulation were markedly decreased by the Tpl2 inhibitor in a dose-dependent fashion (Fig. 2B). These results clearly indicate that the Tp12 inhibitor suppresses c-Fos and NFATc1 gene activation at the transcriptional and translational levels. To investigate whether the above findings also hold true for bone marrow cells, the same experiments were attempted with these cells. As shown in Fig. 3, similar results were obtained with bone marrow precursors. The osteoclastogenesis in bone marrow cells was one-order more potently impaired by the inhibitor than that in RAW264.7 cells.

The role of the MEK/ERK pathway in osteoclastogenesis is controversial. Hotokezaka et al. reported that MEK inhibitors enhanced osteoclastogenesis, ${ }^{18)}$ while Breitkreutz et $a l$. found that an orally available MEK $1 / 2$ inhibitor inhibited osteoclastogenesis in human multiple myeloma through down-regulation of ERK1/2 phosphorylation and c-Fos and NFATc1 gene expression. ${ }^{19)}$ Our present study supports the results of Breitkreutz et al. It is accepted that up-regulation of NFATc1 results in osteoclast differentiation after RANKL stimulation. ${ }^{8,9)}$ c-Fos protein is known to bind to the promoter of the NFATc1 gene in RANKL-induced osteoclast precursors. $^{20)}$ Indeed, RANKL-induced NFATc1 expression is abolished in c-Fos-deficient osteoclast precursors. ${ }^{8,10)}$ Price et $a l^{21)}$ and Murphy et $a{ }^{22}{ }^{22}$ reported that ERK activity is obligatory for stabilizing c-Fos function. Our present results are in excellent agreement with these findings. 
A

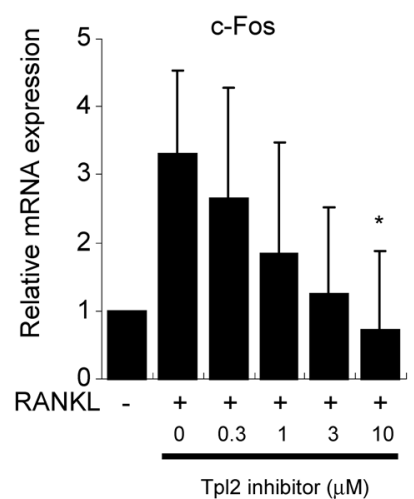

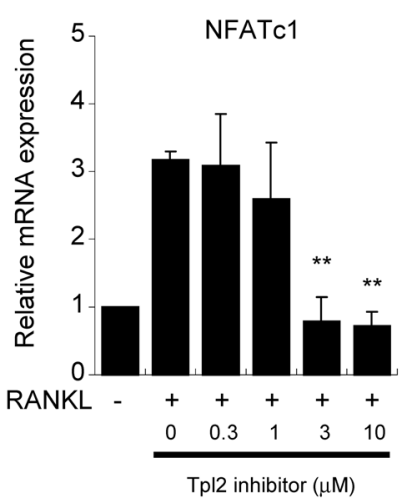

B

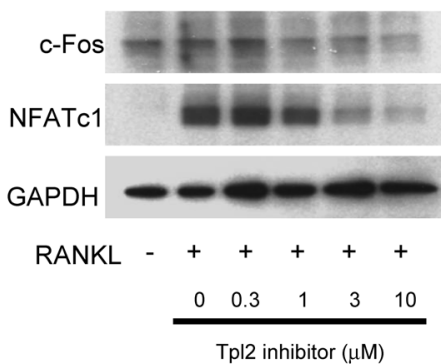

Fig. 2. Suppression of the RANKL-Induced Expression of the Transcription Factors c-Fos and NFATc1 by Tp12 Inhibitor

(A) RAW264.7 cells were incubated with RANKL $(100 \mathrm{ng} / \mathrm{ml})$ and various concentrations of the Tp12 inhibitor for $24 \mathrm{~h}$. Total RNA was isolated from these cells, and c-Fos and NFATc1 mRNA levels were quantified by quantitative RT-PCR. Values are shown as means \pm S.D. of three independent experiments. $* p<0.05$, and $* * p<0.001$ compared with the results of the RANKL-treated cells without Tp12 inhibitor, according to the Students' $t$-test. (B) RAW264.7 cells were incubated with RANKL (100 ng/ml) and various concentrations of the Tpl2 inhibitor for $48 \mathrm{~h}$. The cells were disrupted, and aliquots thereof were subjected to Western blotting as described in Materials and Methods. GAPDH, glyceraldehyde-3-phosphate dehydrogenase.

A

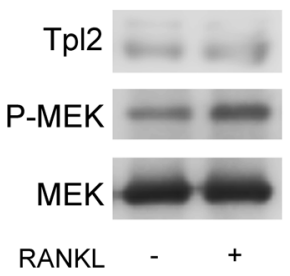

$\mathrm{D}$

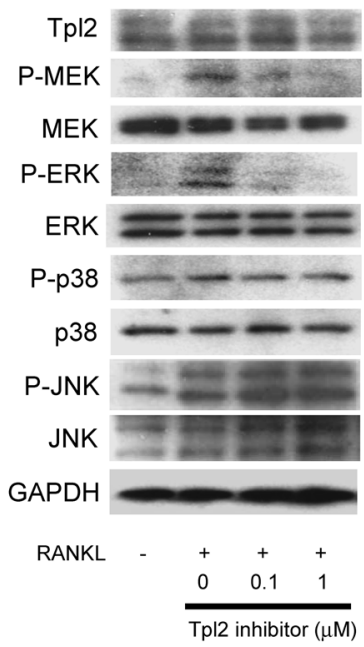

B
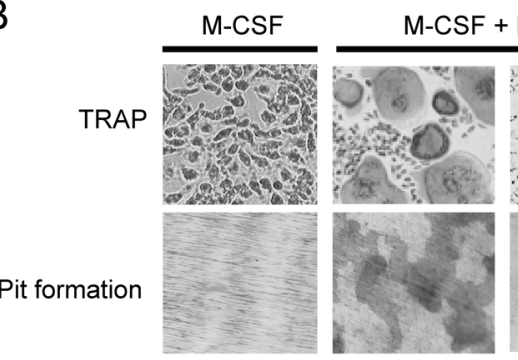

0

Tpl2 inhibitor $(\mu \mathrm{M})$

$\mathrm{E}$
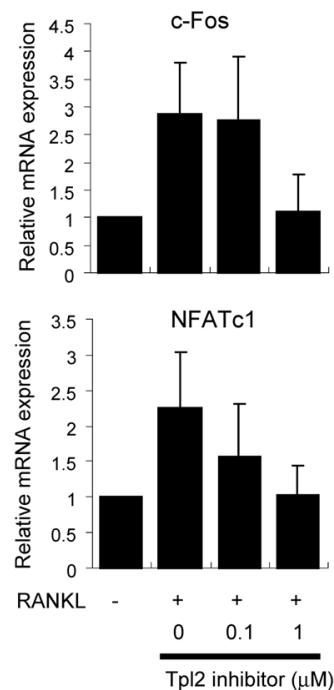

C

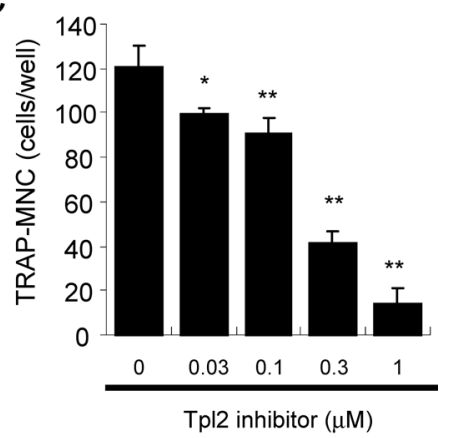

$\mathrm{F}$

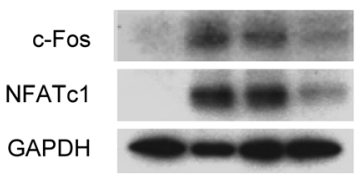

RANKL - $++\quad+$

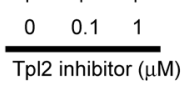

Fig. 3. Suppression of M-CSF and RANKL-Induced Osteoclastogenesis in Murine Bone Marrow Precursors by Tpl2 Inhibitor

(A) Bone marrow precursors were stimulated with RANKL $(100 \mathrm{ng} / \mathrm{ml})$ for $30 \mathrm{~min}$, and then the Tpl2 kinase activity was measured with MEK as the substrate, as described in Materials and Methods. (B) Bone marrow precursors were differentiated into osteoclasts in the presence or absence of the Tpl2 inhibitor as described in Materials and Methods. The cells were subjected to TRAP staining (upper panel) or were subjected to the pit formation assay on dentin slices (lower panel). (C) TRAP-positive multinuclear cells (TRAPMNCs) were quantified. Values are shown as means \pm S.D. of three independent experiments. $* p<0.05$, and $* * p<0.001$ compared with the results in the absence of the inhibitor, according to the Students' $t$-test. (D) The bone marrow precursors were cultured in M-CSF ( $10 \mathrm{ng} / \mathrm{ml})$ for $2 \mathrm{~d}$, and then the cells were incubated with or without varying concentrations of the Tpl2 inhibitor for $1 \mathrm{~h}$, before being exposed to RANKL ( $100 \mathrm{ng} / \mathrm{ml})$ for an additional $30 \mathrm{~min}$. Each protein was assigned by Western blot analysis as described in Materials and Methods. (E) The bone marrow precursors were cultured in M-CSF (10 ng/ml) for $2 \mathrm{~d}$, and then the cells were incubated with or without varying concentrations of the Tpl2 inhibitor and RANKL ( $100 \mathrm{ng} / \mathrm{ml})$ for $24 \mathrm{~h}$, before total RNA was isolated. NFATc1 and c-Fos mRNA levels were determined by quantitative RT-PCR analysis. Values are shown as means \pm S.D. of three independent experiments. (F) The bone marrow precursors were cultured in M-CSF (10 ng/ml) for $2 \mathrm{~d}$, and then the cells were incubated with or without varying concentrations of the Tpl2 inhibitor and RANKL $(100 \mathrm{ng} / \mathrm{ml})$ for $48 \mathrm{~h}$. The c-Fos and NFATc1 protein levels were visualized by Western blot analysis. 
The Tpl2 inhibitor at concentrations of 10 and $1 \mu \mathrm{M}$ was effective at suppressing osteoclast differentiation from RAW264.7 cells and primary bone marrow cells, respectively (Figs. 1, 3). The difference in the sensitivity of these cells to the Tpl 2 inhibitor may be due to various differences in drug uptake, cell metabolism, speed of cell proliferation, mode of cell cycle, and so on. It remains to be clarified whether this inhibitor is also effective in vivo. Furthermore, whether a loss-of-function of Tp12/Cot induced by the introduction of anti-sense RNA would inhibit RANKL-induced osteoclastogenesis remains to be elucidated. It is also of interest to examine whether a gain-of-function of Tpl2/Cot would accelerate RANKL-induced osteoclastogenesis.

Acknowledgment We thank Dr. Hiroaki Sakurai at the University of Toyama and Dr. Naoyuki Takahashi at the Matsumoto Dental University for their kind gifts of antibodies and dentin slices, respectively.

\section{REFERENCES AND NOTES}

1) Present address: Pharmacology Laboratory, Research Division, Mitsubishi Tanabe Pharma Co., 1000 Kamoshida-cho, Aoba-ku, Yokohama, Kanagawa 227-0033, Japan.

2) Present address: Hiroshima University Hospital, Hiroshima 734-8551, Japan.

3) Harris Jr. E. D., N. Engl. J. Med., 322, 1277-1289 (1990).

4) Redlich K., Hayer S., Ricci R., David J. P., Tohidast-Akrad M., Kollias G., Steiner G., Smolen J. S., Wagner E. F., Schett G., J. Clin. Invest., 110, 1419-1427 (2002).

5) Boyce B. F., Xing L., Arch. Biochem. Biophys., 473, 139-146 (2008).

6) Asagiri M., Takayanagi H., Bone, 40, 251-264 (2007).

7) Tanaka S., Nakamura K., Takahasi N., Suda T., Immunol. Rev., 208, 30-49 (2005).

8) Takayanagi H., Kim S., Koga T., Nishina H., Isshiki M., Yoshida H., Saiura A., Isobe M., Yokochi T., Inoue J., Wagner E. F., Mak T. W., Kodama T., Taniguchi T., Dev. Cell, 3, 889—901 (2002).
9) Ishida N., Hayashi K., Hoshijima M., Ogawa T., Koga S., Miyatake Y., Kumegawa M., Kimura T., Takeya T., J. Biol. Chem., 277, 4114741156 (2002).

10) Patriotis C., Makris A., Bear S. E., Tsichlis P. N., Proc. Natl. Acad. Sci. U.S.A., 90, 2251-2255 (1993).

11) Dumitru C. D., Ceci J. D., Tsatsanis C., Kontoyiannis D., Stamatakis K., Lin J. H., Patriotis C., Jenkins N. A., Copeland N. G., Kollias G., Tsichlis P. N., Cell, 103, 1071-1083 (2000).

12) Kontoyiannis D., Boulougouris G., Manoloukos M., Armaka M., Apostolaki M., Pizarro T., Kotlyarov A., Forster I., Flavell R., Gaestel M., Tsichlis P., Cominelli F., Kollias G., J. Exp. Med., 196, 1563 1574 (2002).

13) Van Acker G. J., Perides G., Weiss E. R., Das S., Tsichlis P. N., Steer M. L., J. Biol. Chem., 282, 22140-22149 (2007).

14) Gavrin L. K., Green N., Hu Y., Janz K., Kaila N., Li H. Q., Tam S. Y., Thomason J. R., Gopalsamy A., Ciszewski G., Cuozzo J. W., Hall J. P., Hsu S., Telliez J. B., Lin L. L., Bioorg. Med. Chem. Lett., 15, 52885292 (2005).

15) Beinke S., Deka J., Lang V., Belich M. P., Walker P. A., Howell S., Smerdon S. J., Gamblin S. J., Ley S. C., Mol. Cell. Biol., 23, 47394752 (2003).

16) Kamel Mohamed S. G., Sugiyama E., Shinoda K., Hounoki H., Taki H., Maruyama M., Miyahara T., Kobayashi M., Biochem. Biophys. Res. Commun., 329, 839-845 (2005).

17) Hall J. P., Kurdi Y., Hsu S., Cuozzo J., Liu J., Telliez J. B., Seidl K. J., Winkler A., Hu Y., Green N., Askew G. R., Tam S., Clark J. D., Lin L. L., J. Biol. Chem., 282, 33295-33304 (2007).

18) Hotokezaka H., Sakai E., Kanaoka K., Saito K., Matsuo K., Kitaura H., Yoshida N., Nakayama K., J. Biol. Chem., 277, 47366-47372 (2002).

19) Breitkreutz I., Raab M. S., Vallet S., Hideshima T., Raje N., Chauhan D., Munshi N. C., Richardson P. G., Anderson K. C., Br. J. Haematol., 139, 55-63 (2007).

20) Matsuo K., Galson D. L., Zhao C., Peng L., Laplace C., Wang K. Z., Bachler M. A., Amano H., Aburatani H., Ishikawa H., Wagner E. F., J. Biol. Chem., 279, 26475-26480 (2004).

21) Price M. A., Cruzalegui F. H., Treisman R., EMBO J., 15, 6552-6563 (1996).

22) Murphy L. O., Smith S., Chen R. H., Fingar D. C., Blenis J., Nat. Cell Biol., 4, 556-564 (2002). 